\title{
On the Exponential Stability of Moving Horizon Observer for Globally $\mathrm{N}$-Detectable Nonlinear Systems
}

\author{
Dan Sui and Tor Arne Johansen ${ }^{\dagger}$
}

\begin{abstract}
A moving horizon observer is analyzed for nonlinear discrete-time systems. Exponential stability relies on a global detectability assumption that utilizes the concept of incremental input-to-state-stability.
\end{abstract}

Key Words: Estimation, Detectability, Incremental Input-to-state-stability

\section{INTRODUCTION}

Consider the discrete-time nonlinear system:

$$
\begin{aligned}
x(t+1) & =f(x(t), u(t)) \\
y(t) & =h(x(t), u(t)),
\end{aligned}
$$

where $x(t) \in R^{n_{x}}, u(t) \in R^{n_{u}}$ and $y(t) \in R^{n_{y}}$ are respectively the state, input and measurement vectors, and $t$ is the discrete time index.

A Moving Horizon State Estimator (MHE) makes use of a finite memory moving window of both current and recent measurement data in a least-squares criterion, possibly in addition to a state estimate and covariance matrix estimate to set the initial cost at the beginning of the data window, see $[1,2,3]$ for various formulations of the problem. Slightly different formulations of the uniform observability assumption are used in these references for the stability or convergence proofs. Uniform observability essentially means that every input sequence will excite the system to the extent that the state can be computed from the information in the input and output data. This is a restrictive assumption that is likely not to hold in certain interesting and important state estimation applications, in particular combined state and parameter estimation problems where the input may not be persistently

\footnotetext{
Department of Engineering Cybernetics, Norwegian University of Science and Technology, Trondheim, Norway. *

This work was supported by the Research Council of Norway under the Strategic University Program on Computational Methods in Nonlinear Motion Control and the Center for Integrated Operations in the Petroleum Industry.

${ }^{*} \mathrm{D}$. Sui is currently with SINTEF.

tor.arne.johansen@itk.ntnu.no
}

exciting. Hence, a regularized moving horizon observer that degrades gracefully in the lack of excitations was introduced in $[4,5]$.

In $[4,5]$, strongly detectable systems [6] are considered, and convergence on compact sets is analyzed. The novel contribution of the present work is to relax the strong detectability conditions by using the concept of incremental input-to-state stability [7] in order to provide global exponential stability conditions.

Methods for regularization to ensure graceful degradation of performance in cases when the input is not $\mathrm{N}$-exciting can be derived based on the present stability condition by following a similar technique as in $[4,5]$. It is therefore not considered here.

The following notation and nomenclature is used. $\|x\|_{A}^{2}=x^{T} A x$ by $A \geq 0$. For two vectors $x \in R^{n}$ and $y \in R^{m}$ we let $\operatorname{col}(x, y)$ denote the column vector in $R^{n+m}$ where $x$ and $y$ are stacked into a single column. The composition of two functions $f$ and $g$ is written $f \circ g(x)=f(g(x))$. A function $\varphi: R^{+} \rightarrow R^{+}$is called a $K$-function if $\varphi(0)=0$ and it is strictly increasing. A function $\varphi: R^{+} \rightarrow R^{+}$is called a $K_{\infty}$-function if $\varphi \in K$ and it is radially unbounded. A function $\beta$ : $R^{+} \times R^{+} \rightarrow R^{+}$is called a $K L$-function if for each fixed $k \in R^{+}, \beta(\cdot, k) \in K$ and for each fixed $s \in R^{+}, \beta(s, \cdot)$ is non-increasing and $\lim _{k \rightarrow \infty} \beta(s, k)=0$. For a sequence $\{z(j)\}$ for $j \geq 0, z_{[t]}$ denotes the truncation of $\{z(j)\}$ at time $t$, i.e. $z_{[t]}=\{z(j)\}$ for $0 \leq j \leq t$. 


\section{Nonlinear MHE formulation}

The $N+1$ consecutive measurements of outputs and inputs until time $t$ are denoted as

$$
Y_{t}=\left[\begin{array}{r}
y(t-N) \\
y(t-N+1) \\
\vdots \\
y(t)
\end{array}\right], \quad U_{t}=\left[\begin{array}{r}
u(t-N) \\
u(t-N+1) \\
\vdots \\
u(t)
\end{array}\right] .
$$

Define the $N$-information vector at time $t$ as

$$
I(t)=\operatorname{col}(y(t-N), \ldots, y(t), u(t-N), \ldots, u(t)) .
$$

To express $Y_{t}$ as a function of $x(t-N)$ and $U_{t}$, denote $f^{u(t)}(x(t))=f(x(t), u(t))$ and $h^{u(t)}(x(t))=$ $h(x(t), u(t))$, and note from (1b) that the following algebraic map can be formulated [2]:

$$
\begin{aligned}
Y_{t} & =H\left(x(t-N), U_{t}\right)=H_{t}(x(t-N)) \\
& =\left[\begin{array}{r}
h^{u(t-N)}(x(t-N)) \\
h^{u(t-N+1)} \circ f^{u(t-N)}(x(t-N)) \\
\vdots \\
h^{u(t)} \circ f^{u(t-1)} \circ \cdots \circ f^{u(t-N)}(x(t-N))
\end{array}\right] .
\end{aligned}
$$

The proposed MHE problem consists in estimating, at any time $t=N, N+1, \ldots$, the state vectors $x(t-$ $N), \ldots, x(t)$, on the basis of a priori estimates $\bar{x}(t-N)$ and the information vector $I(t)$. A convergent estimator is pursued by minimizing the following weighted regularized least-squares criterion

$$
\begin{aligned}
J(\hat{x}(t-N \mid t) ; \bar{x}(t-N), I(t)) & =\left\|Y_{t}-H\left(\hat{x}(t-N \mid t), U_{t}\right)\right\|_{W_{t}}^{2} \\
& +\|\hat{x}(t-N \mid t)-\bar{x}(t-N)\|_{M}^{2}
\end{aligned}
$$

with $M \geq 0$ and $W_{t} \geq 0$ being symmetric time-varying weight matrices, and $\hat{x}(t-N \mid t)$ denotes any estimate of $x(t-N)$ based on information up to time $t$. In particular, let $J_{t}^{o}=\min _{\hat{x}(t-N \mid t)} J(\hat{x}(t-N \mid t) ; \bar{x}(t-N), I(t))$, and $\hat{x}^{o}(t-N \mid t)$ be the associated optimal estimate. The a priori estimator is determined from the previous optimal estimate $\hat{x}^{o}(t-N-1 \mid t-1)$, that is,

$$
\bar{x}(t-N)=f\left(\hat{x}^{o}(t-N-1 \mid t-1), u(t-N-1)\right) .
$$

Before we introduce the main assumptions and stability analysis of the dynamics of the estimation error

$$
e(t-N)=x(t-N)-\hat{x}^{o}(t-N \mid t)
$$

we need to introduce the concept of incrementally input-to-state stablility.

\section{Incremental input-to-state-stability}

We define the notion of global incremental inputto-state stability $(\delta$ ISS) [7].

Definition 1 The system (1a) is globally $\delta I S S$, if there exist a KL-function $\theta$ and a $K_{\infty}$-function $\gamma_{u}$ such that for any $t \geq 0$, any initial conditions $x(0), \bar{x}(0) \in R^{n_{x}}$ and any $u_{[t-1]}, \bar{u}_{[t-1]}$ with $u(j), \bar{u}(j) \in R^{n_{u}}, 0 \leq j \leq t-1$, the following is true:

$$
\begin{aligned}
\|x(t)-\bar{x}(t)\| \leq & \theta(\|x(0)-\bar{x}(0)\|, t) \\
& +\gamma_{u}\left(\left\|u_{[t-1]}-\bar{u}_{[t-1]}\right\|\right) .
\end{aligned}
$$

Definition 2 ( $\delta I S S$-Lyapunov Function) A continuous function $V: R^{n_{x}} \times R^{n_{x}} \rightarrow R \geq 0$ is called a $\delta I S S$ Lyapunov function for the system (1a) if the following holds:

1. $V(0,0)=0$.

2. There exist $K_{\infty}$-functions $\alpha_{1}, \alpha_{2}$ such that for any $x, \bar{x}$,

$$
\alpha_{1}(\|x-\bar{x}\|) \leq V(x, \bar{x}) \leq \alpha_{2}(\|x-\bar{x}\|) .
$$

3. There exists a K-function $\sigma$, such that for any $x, \bar{x}$ and any couple of input signals $u, \bar{u}$

$$
\begin{aligned}
V(f(x, u), f(\bar{x}, \bar{u}))-V(x, \bar{x}) \leq & -\alpha_{3}(\|x-\bar{x}\|) \\
& +\sigma(\|u-\bar{u}\|)
\end{aligned}
$$

with $\alpha_{3}$ positive on $R^{+}$.

The proof of the following result is similar to [8]:

Theorem 1 If there exists a $\delta I S S$-Lyapunov function for (1a), then the system (1a) is SISS. Moreover, the $\delta I S S$ property holds for some $\rho \in[0,1)$ with

$$
\theta(s, t)=\alpha_{1}^{-1}\left(2 \rho^{t} \alpha_{2}(s)\right), \quad \gamma_{u}(s)=\alpha_{1}^{-1}\left(\frac{2 \sigma(s)}{1-\rho}\right),
$$

Definition 3 (Quadratic $\delta I S S$-Lyapunov Function) A continuous function $V(x, \bar{x}, P)=\|x-\bar{x}\|_{P}^{2}$ with $P=$ $P^{T}>0$ is called a quadratic $\delta I S S$-Lyapunov function for the system (1a) if the following holds:

1. $V(0,0, P)=0$.

2. There exist a symmetric matrix $Q>0$ and $a$ symmetric matrix $\Delta>0$, such that for any $x, \bar{x}$ and any couple of input signals $u, \bar{u}$,

$$
\begin{aligned}
V(f(x, u), f(\bar{x}, \bar{u}), P)-V(x, \bar{x}, P) \leq & -V(x, \bar{x}, Q) \\
& +V(u, \bar{u}, \Delta) .
\end{aligned}
$$


Lemma 1 Consider the system (1a) with $f$ globally Lipschitz and continuously differentiable. The system has a quadratic $\delta I S S-L y a p u n o v$ function $V$ with a symmetric matrix $Q>0$ and a symmetric matrix $\Delta>0$ and a Lyapunov matrix $P=P^{T}>0$ if for all $x, \bar{x} \in R^{n_{x}}$ and $u \in R^{n_{u}}$

$$
2 \Lambda^{T}(x, \bar{x}) P \Lambda(x, \bar{x})-P \leq-Q,
$$

for some symmetric $Q>0$ and $\Lambda(x, \bar{x})=\int_{0}^{1} \frac{\partial}{\partial x} f((1-$ $s) x+s \bar{x}, u) d s$.

Proof. Consider any two states $x, \bar{x}$ and two inputs $u, \bar{u}$. It is easy to obtain

$$
\begin{aligned}
& f(x, u)-f(\bar{x}, \bar{u}) \\
& =f(x, u)-f(\bar{x}, u)+f(\bar{x}, u)-f(\bar{x}, \bar{u}) .
\end{aligned}
$$

Then we know that

$$
\begin{aligned}
& V(f(x, u), f(\bar{x}, \bar{u}), P) \\
& \leq 2 V(f(x, u), f(\bar{x}, u), P)+2 V(f(\bar{x}, u), f(\bar{x}, \bar{u}), P) .
\end{aligned}
$$

From Proposition 2.4.7 in [9], we have

$$
\begin{aligned}
& f(x, u)-f(\bar{x}, u)=\Lambda(x, \bar{x})(x-\bar{x}), \\
& f(\bar{x}, u)-f(\bar{x}, \bar{u})=\Theta(u, \bar{u})(u-\bar{u}),
\end{aligned}
$$

where $\Theta(u, \bar{u})=\int_{0}^{1} \frac{\partial}{\partial u} f(\bar{x},(1-s) u+s \bar{u}) d s$. Inequality (12) becomes

$$
\begin{aligned}
& V(f(x, u), f(\bar{x}, \bar{u}), P) \leq 2 V\left(x, \bar{x}, \Lambda^{T}(x, \bar{x}) P \Lambda(x, \bar{x})\right) \\
& +2 V\left(u, \bar{u}, \Theta^{T}(u, \bar{u}) P \Theta(u, \bar{u})\right) .
\end{aligned}
$$

Since (11) holds, it implies that

$$
2 V\left(x, \bar{x}, \Lambda^{T}(x, \bar{x}) P \Lambda(x, \bar{x})\right)-V(x, \bar{x}, P)<-V(x, \bar{x}, Q) .
$$

There always exits $\Delta$ such that $\Delta>2 \Theta^{T}(u, \bar{u}) P \Theta(u, \bar{u})$ since $\Theta(\cdot)$ is bounded, i.e. $f$ is globally Lipschitz.

\section{Stability analysis}

Definition 4 The system (1a)-(1b) is globally $\mathrm{N}$ observable if there exists a $K$-function $\varphi$ such that for any $x_{1}, x_{2}$ there exists a $U_{t}$ such that

$$
\varphi\left(\left\|x_{1}-x_{2}\right\|^{2}\right) \leq\left\|H\left(x_{1}, U_{t}\right)-H\left(x_{2}, U_{t}\right)\right\|^{2} .
$$

Definition 5 The input $U_{t}$ is said to be $N$-exciting for the globally $N$-observable system (1a)-(1b) at time $t$ if there exists a $K$-function $\varphi_{t}$ such that for any $x_{1}, x_{2}$ satisfying

$$
\varphi_{t}\left(\left\|x_{1}-x_{2}\right\|^{2}\right) \leq\left\|H\left(x_{1}, U_{t}\right)-H\left(x_{2}, U_{t}\right)\right\|^{2} .
$$

When a system is not $N$-observable, it is in general not possible to reconstruct exactly all the state components from the $\mathrm{N}$-information vector. The reason for this is that violation of Def. 4 basically means that the system of algebraic equations $Y_{t}=H\left(x(t-N), U_{t}\right)$ may not be invertible in the sence that it may not have a unique solution $x(t-N)$ for given $Y_{t}, U_{t}$. However, one may be able to reconstruct exactly at least some components of $x(t-N)$, based on the $N$ information vector, and the remaining components can be reconstructed asymptotically. This corresponds to the notion of detectability [6], where we suppose there exists a coordinate transform $T: R^{n_{x}} \rightarrow R^{n_{x}}$

$$
d=\left(\begin{array}{l}
\xi \\
z
\end{array}\right)=T(x)
$$

that leads to the following form

$$
\begin{aligned}
\xi(t+1) & =F_{1}(\xi(t), z(t), u(t)) \\
z(t+1) & =F_{2}(z(t), u(t)) \\
y(t) & =g(z(t), u(t)) .
\end{aligned}
$$

This transform effectively partitions the state $x$ into an observable sub-state $z \in R^{n_{z}}$ and an unobservable substate $\xi \in R^{n_{\xi}}$, and the following global detectability definition can be given:

Definition 6 The system $(1 a)-(1 b)$ is globally $N$ detectable if

1. There exists a coordinate transform $T$ that brings the system in the form $(14 a)-(14 c)$.

2. The sub-system (14b)-(14c) is globally $N$ observable.

3. The sub-system (14a) has a quadratic $\delta I S S$ Lyapunov function.

Definition 7 The input $U_{t}$ is said to be $N$-exciting for a globally $\mathrm{N}$-detectable system (1a)-(1b) at time $t$ if it is $N$-exciting for the associated globally $N$-observable sub-system (14b)-(14c) at time $t$.

The following regularity properties are assumed throughout this paper:

(A1) The functions $f$ and $h$ are globally Lipschitz and twice differentiable.

(A2) The function $T$ is continuously differentiable, globally Lipschitz and bounded away from singularity for all $x \in R^{n_{x}}$ such that $T^{-1}(x)$ is well defined. It is also assumed that $T^{-1}(x)$ is globally Lipschitz.

(A3) The system (1a)-(1b) is globally $N$-detectable and the input $U_{t}$ is $N$-exciting for all $t \geq 0$. Moreover, 
the sub-system (14a) has a quadratic $\delta I S S$-Lyapunov function $V\left(\xi_{1}, \xi_{2}, P_{\xi}\right)$ such that $P_{\xi}=P_{\xi}^{T}>0$ with symmetric matrices $Q_{\xi}>0$ and $\Delta_{z}>0, \Delta_{u}>0$, that is,

$$
\begin{aligned}
& V\left(F_{1}\left(\xi_{1}, z_{1}, u_{1}\right), F_{1}\left(\xi_{2}, z_{2}, u_{2}\right), P_{\xi}\right)-V\left(\xi_{1}, \xi_{2}, P_{\xi}\right) \\
& \leq-V\left(\xi_{1}, \xi_{2}, Q_{\xi}\right)+V\left(u_{1}, u_{2}, \Delta_{u}\right)+V\left(z_{1}, z_{2}, \Delta_{z}\right) .
\end{aligned}
$$

(A4) $x(t), u(t)$ and $y(t)$ are bounded for all $t \geq 0$.

In the stability analysis we will need to make use of the coordinate transform into observable and unobservable states, although we emphasize that knowledge of this transform is not needed for the implementation of the observer.

Theorem 2 Suppose that assumptions (A1)-(A4) hold. Then for any $M \geq 0$, there exists a sufficiently large weight matrix $W_{t} \geq 0$ such that the observer error dynamics is globally exponentially stable.

Proof. To express $Y_{t}$ as a function of $z(t-N)$ and $U_{t}$, the following algebraic mapping can be formulated similar to the mapping $H$ :

$$
\begin{aligned}
Y_{t} & =G\left(z(t-N), U_{t}\right)=G_{t}(z(t-N)) \\
& =\left[\begin{array}{r}
g^{u(t-N)}(z(t-N)) \\
g^{u(t-N+1)} \circ F_{2}^{u(t-N)}(z(t-N)) \\
\vdots \\
g^{u(t)} \circ F_{2}^{u(t-1)} \circ \cdots \circ F_{2}^{u(t-N)}(z(t-N))
\end{array}\right] .
\end{aligned}
$$

In order to state the stability result and the proof, the following definitions are given:

$$
\begin{aligned}
\hat{\Phi}_{t} & =\hat{\Phi}_{t}\left(z(t-N), \hat{z}^{o}(t-N \mid t)\right) \\
& =\int_{0}^{1} \frac{\partial}{\partial z} G\left((1-s) z(t-N)+s \hat{z}^{o}(t-N \mid t), U_{t}\right) d s \\
\hat{\Upsilon}_{t} & =\Upsilon_{t}\left(\breve{x}(t-N-1), \hat{x}^{o}(t-N-1 \mid t-1)\right) \\
& =\int_{0}^{1} \frac{\partial}{\partial x} f((1-s) \breve{x}(t-N-1) \\
& \left.+s \hat{x}^{o}(t-N-1 \mid t-1), u(t-N-1)\right) d s, \\
\hat{\Gamma}_{t} & =\Gamma_{t}\left(\breve{d}(t-N-1), \hat{d}^{o}(t-N-1 \mid t-1)\right) \\
& =\int_{0}^{1} \frac{\partial}{\partial d} T^{-1}((1-s) \breve{d}(t-N-1) \\
& \left.+s \hat{d}^{o}(t-N-1 \mid t-1)\right) d s,
\end{aligned}
$$

where

$$
\begin{aligned}
& \breve{x}(t-N-1)=T^{-1}(\breve{d}(t-N-1)) \\
& \breve{d}(t-N-1)=\operatorname{col}\left(\hat{\xi}^{o}(t-N-1 \mid t-1), z(t-N-1)\right)
\end{aligned}
$$

and

$$
\begin{aligned}
\hat{d}^{o}(t-N-1 \mid t-1) & =T\left(\hat{x}^{o}(t-N-1 \mid t-1)\right) \\
\left(\begin{array}{c}
\hat{\xi}^{o}(t-N \mid t) \\
\hat{z}^{o}(t-N \mid t)
\end{array}\right) & =T \hat{x}^{o}(t-N \mid t)
\end{aligned}
$$

The basic idea behind the proof consists in looking for upper and lower bounds on the optimal cost $J_{t}^{o}$.

Lower bound on the optimal cost $J_{t}^{o}$

Using the fact that system (1a)-(1b) can be transformed using (13), there exist $d(t-N)=T(x(t-N)), \hat{d}^{o}(t-$ $N \mid t)=T\left(\hat{x}^{o}(t-N \mid t)\right)$ such that in the new coordinates, the system is in the form of (14a)-(14c). Note that the first term in the right-hand side of expression (4) in the new coordinates can be rewritten as

$$
\begin{aligned}
& \left\|Y_{t}-G\left(\hat{z}^{o}(t-N \mid t), U_{t}\right)\right\|_{W_{t}}^{2} \\
& =\left\|G\left(z(t-N), U_{t}\right)-G\left(\hat{z}^{o}(t-N \mid t), U_{t}\right)\right\|_{W_{t}}^{2} .
\end{aligned}
$$

From Proposition 2.4.7 in [9], since (A1) and (A2) hold, we have

$$
\begin{aligned}
& G\left(z(t-N), U_{t}\right)-G\left(\hat{z}^{o}(t-N \mid t), U_{t}\right) \\
& =\hat{\Phi}_{t}\left(z(t-N), \hat{z}^{o}(t-N \mid t)\right)\left(z(t-N)-\hat{z}^{o}(t-N \mid t)\right) .
\end{aligned}
$$

Then we have

$$
\begin{aligned}
& \left\|Y_{t}-G\left(\hat{z}^{o}(t-N \mid t), U_{t}\right)\right\|_{W_{t}}^{2} \\
& \quad=\left\|z(t-N)-\hat{z}^{o}(t-N \mid t)\right\|_{\hat{\Phi}_{t}^{T} W_{t} \hat{\Phi}_{t}}^{2}
\end{aligned}
$$

Taking zero as the lower bound on the second term of (4) we get

$$
J_{t}^{o} \geq\left\|z(t-N)-\hat{z}^{o}(t-N \mid t)\right\|_{\hat{\Phi}_{t}^{T} W_{t} \hat{\Phi}_{t}}^{2} .
$$

Upper bound on the optimal cost $J_{t}^{o}$

Let $\breve{x}(t-N)=f(\breve{x}(t-N-1), u(t-N-1))$. From the optimality of $\hat{x}^{o}(t-N \mid t)$, we have $J_{t}^{o} \leq J(\breve{x}(t-N) ; \bar{x}(t-$ $N), I(t))$. Combining the upper and lower bound on $J_{t}^{o}$,

$$
\begin{aligned}
& J(\breve{x}(t-N) ; \bar{x}(t-N), I(t)) \\
& \quad \geq\left\|z(t-N)-\hat{z}^{o}(t-N \mid t)\right\|_{\hat{\Phi}_{t}^{T} W_{t} \hat{\Phi}_{t}}^{2}
\end{aligned}
$$

Consider the cost function $J(\breve{x}(t-N) ; \bar{x}(t-N), I(t))$. Since the output of the system (14) must equal the output of the system (1) for equivalent initial states and equal input, we have from (18) and (19) that $\left\|Y_{t}-H\left(\breve{x}(t-N), U_{t}\right)\right\|_{W_{t}}^{2}=\| G\left(z(t-N), U_{t}\right)-G(z(t-$ $\left.N), U_{t}\right) \|_{W_{t}}^{2}=0$. Also, from Proposition 2.4.7 in [9],

$$
\begin{aligned}
& \breve{x}(t-N)-\bar{x}(t-N) \\
& \quad=\hat{\Upsilon}_{t}\left(\breve{x}(t-N-1)-\hat{x}^{o}(t-N-1 \mid t-1)\right),
\end{aligned}
$$


and

$$
\begin{aligned}
& \breve{x}(t-N-1)-\hat{x}^{o}(t-N-1 \mid t-1) \\
& =\hat{\Gamma}_{t}\left(\breve{d}(t-N-1)-\hat{d}^{o}(t-N-1 \mid t-1)\right) \\
& =\hat{\Gamma}_{t}\left[\begin{array}{c}
\hat{\xi}^{o}(t-N-1 \mid t-1)-\hat{\xi}^{o}(t-N-1 \mid t-1) \\
z(t-N-1)-\hat{z}^{o}(t-N-1 \mid t-1)
\end{array}\right] \\
& =\hat{\Gamma}_{t} \eta^{T}\left(z(t-N-1)-\hat{z}^{o}(t-N-1 \mid t-1)\right),
\end{aligned}
$$

where $\eta=\left[\mathbf{0}_{n_{z} \times n_{\xi}}, I_{n_{z} \times n_{z}}\right]$. Let $\Omega_{t}=\hat{\Gamma}_{t} \eta^{T}$. We have

$$
\begin{aligned}
& \|\breve{x}(t-N)-\bar{x}(t-N)\|_{M}^{2} \\
& =\left\|z(t-N-1)-\hat{z}^{o}(t-N-1 \mid t-1)\right\|_{\Omega_{t}^{T} \hat{\Upsilon}_{t}^{T} M \hat{\Upsilon}_{t} \Omega_{t}}^{2}
\end{aligned}
$$

Therefore,

$$
\begin{aligned}
& \left\|z(t-N)-\hat{z}^{o}(t-N \mid t)\right\|_{\hat{\Phi}_{t}^{T} W_{t} \hat{\Phi}_{t}}^{2} \\
& \leq\left\|z(t-N-1)-\hat{z}^{o}(t-N-1 \mid t-1)\right\|_{\Omega_{t}^{T} \hat{\Upsilon}_{t}^{T} M \hat{\Upsilon}_{t} \Omega_{t}}^{2} .
\end{aligned}
$$

Proof of the stability.

Consider a Lyapunov function

$$
V(s(t))=\left\|s_{1}(t)\right\|_{P_{1}}^{2}+\left\|s_{2}(t)\right\|_{P_{2}}^{2},
$$

where $s(t)=\operatorname{col}\left(s_{1}(t), s_{2}(t)\right), P_{1}>0$ and $P_{2}=P_{\xi}\left(P_{\xi}\right.$ is given in (10)) for all $t \geq 0$. Let

$$
\begin{aligned}
& s_{1}(t)=z(t-N)-\hat{z}^{o}(t-N \mid t), \\
& s_{2}(t)=\xi(t-N)-\hat{\xi}^{o}(t-N \mid t) .
\end{aligned}
$$

In the following $V(s(t))-V(s(t-1))<0, \forall s(t) \neq 0$ for some $W_{t}$ is shown.

$$
\begin{aligned}
& V(s(t))-V(s(t-1)) \\
& =\left\|s_{1}(t)\right\|_{P_{1}}^{2}-\left\|s_{1}(t-1)\right\|_{P_{1}}^{2}+\left\|s_{2}(t)\right\|_{P_{2}}^{2}-\left\|s_{2}(t-1)\right\|_{P_{2}}^{2} .
\end{aligned}
$$

Notice that the first term of (4) does not depend on $\hat{\xi}(t-N \mid t)$. Hence $\hat{\xi}^{o}(t-N \mid t)$ is completely determined by the minimization of the second term of (4), leading to $\hat{\xi}^{o}(t-N \mid t)=\bar{\xi}(t-N)$. Since (A3) holds, then there exists a quadratic $\delta I S S$-Lyapunov function such that (10) is true. Then,

$$
\begin{aligned}
\left\|s_{2}(t)\right\|_{P_{2}}^{2}-\left\|s_{2}(t-1)\right\|_{P_{2}}^{2} \leq & -\left\|s_{2}(t-1)\right\|_{Q_{\xi}}^{2} \\
& +\left\|s_{1}(t-1)\right\|_{\Delta_{z}}^{2} .
\end{aligned}
$$

Therefore, we know that

$$
\begin{aligned}
V(s(t))-V(s(t-1)) & \leq-\left\|s_{2}(t-1)\right\|_{Q_{\xi}}^{2}+\left\|s_{1}(t)\right\|_{P_{1}}^{2} \\
& -\left\|s_{1}(t-1)\right\|_{P_{1}}^{2}+\left\|s_{1}(t-1)\right\|_{\Delta_{z}}^{2} .
\end{aligned}
$$

Since (A1)-(A4) hold, $\hat{\Phi}_{t}$ has full rank and $\left\|\hat{\Phi}_{t}^{T} \hat{\Phi}_{t}\right\| \geq$ $\varepsilon I$ for some $\varepsilon>0$, there always exists $W_{t}$ such that

$$
\hat{\Phi}_{t}^{T} W_{t} \hat{\Phi}_{t} \geq P_{1} .
$$

It follows that

$$
\left\|s_{1}(t)\right\|_{P_{1}}^{2} \leq\left\|s_{1}(t)\right\|_{\hat{\Phi}_{t}^{T} W_{t} \hat{\Phi}_{t}}^{2} \leq\left\|s_{1}(t-1) \mid\right\|_{\Omega_{t}^{T} \hat{\Upsilon}_{t}^{T} M \hat{\Upsilon}_{t} \Omega_{t}}^{2} .
$$

Then, we have

$$
\begin{aligned}
& \left\|s_{1}(t)\right\|_{P_{1}}^{2}-\left\|s_{1}(t-1)\right\|_{P_{1}}^{2}+\left\|s_{1}(t-1)\right\|_{\Delta_{z}}^{2} \leq \\
& \left\|s_{1}(t-1)\right\|_{\Omega_{t}^{T} \hat{\Upsilon}_{t}^{T} M \hat{\Upsilon}_{t} \Omega_{t}}^{2}-\left\|s_{1}(t-1)\right\|_{P_{1}}^{2}+\left\|s_{1}(t-1)\right\|_{\Delta_{z}}^{2} .
\end{aligned}
$$

Since $\left\|\hat{\Upsilon}_{t}\right\|$ and $\left\|\hat{\Gamma}_{t}\right\|$ are bounded, there always exists a sufficiently large weight matrix $W_{t}$ such that for all $t \geq 0$

$$
\begin{aligned}
\hat{\Phi}_{t}^{T} W_{t} \hat{\Phi}_{t} & \geq P_{1} \\
P_{1} & \geq \Omega_{t}^{T} \hat{\Upsilon}_{t}^{T} M \hat{\Upsilon}_{t} \Omega_{t}+\Delta_{z}+\tilde{\Delta} \\
W_{t} & \geq 0
\end{aligned}
$$

for some symmetric $\tilde{\Delta}>0$. Then we have

$V(s(t))-V(s(t-1)) \leq-\left\|s_{1}(t-1)\right\|_{\tilde{\Delta}}^{2}-\left\|s_{2}(t-1)\right\|_{Q_{\xi}}^{2}$,

which implies that $s(t)$ is globally exponentially stable. Since (A2) holds, it is easy to obtain that the error dynamics is globally exponentially stable.

\section{Numerical example}

Consider the following nonlinear system

$$
\begin{aligned}
\dot{x}_{1} & =-4 x_{1}+x_{2} \\
\dot{x}_{2} & =-x_{2}+x_{3} u \\
\dot{x}_{3} & =0 \\
y & =x_{2}+v .
\end{aligned}
$$

It is clear that $x_{1}$ is not observable, but corresponds to a $\delta$ ISS system. It is also clear that the observability of $x_{3}$ will depend on the excitation $u$, while $x_{2}$ is generally observable. One may think of $x_{3}$ as a parameter representing an unknown gain on the input, where the third state equation is an augmentation for the purpose of estimating this parameter.

The same observability and detectability properties hold for the discretized system with sampling interval $t_{f}=0.1$. It is easy to know that the sub-system (28a) has a quadratic $\delta$ ISS-Lyapunov function with $P_{\xi}=1$ and $\Delta_{z}=\left[\begin{array}{cc}0.02, & 0 \\ 0, & 0.01\end{array}\right]$. 
In this example, $W_{t}, M$ are chosen as $W_{t}=$ $\alpha I_{N n_{y}}, M=\beta I_{n_{x}}$. The problem becomes to find suitable $\alpha, \beta$ such that (26) holds.

In this simulation example we choose $x_{0}=$ $[4,-7,2], \bar{x}_{0}=[3,-5.9,-1]$, and $N=2$. The input is discrete-time white noise. Choose $\beta=0.01, \alpha=$ 4. The simulation result is shown in Figure 1 when measurement noise is added, independent uniformly distributed $v \in[-0.01,0.01]$. True states are shown in solid line; estimated states of proposed work are shown in dash-dot line.
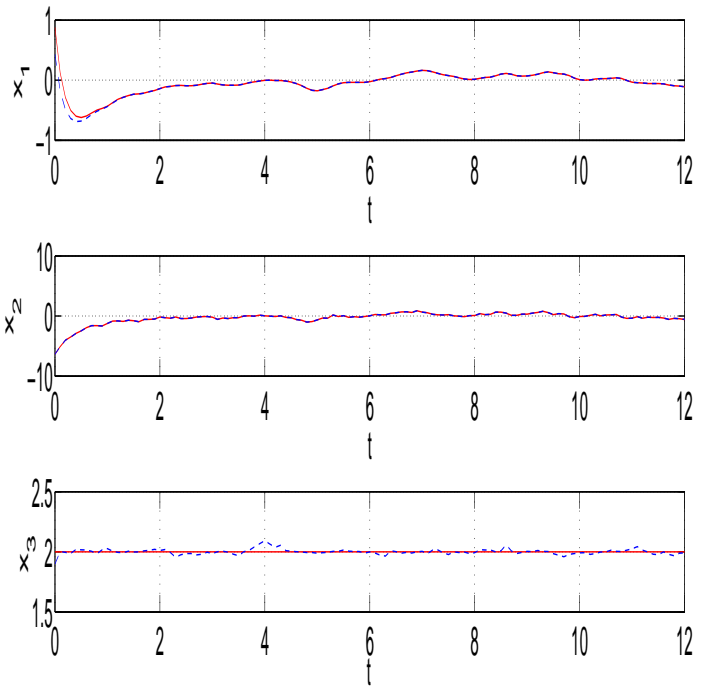

Fig. 1. Simulation results.

\section{Conclusions}

The strong detectability assumption that formed the basis of convergence analysis on compact sets in $[4,5]$ was replaced by a global detectability assumption based on the concept incremental inputto-state-stability. This leds to a global convergence analysis.
2. P. E. Moraal and J. W. Grizzle. Observer design for nonlinear systems with discrete-time measurement. IEEE Transactions Automatic Control, 40:395-404, 1995.

3. A. Alessandri, M. Baglietto, and G. Battistelli. Moving-horizon state estimation for nonlinear discrete-time systems: New stability results and approximation schemes. Automatica, 44:17531765, 2008.

4. D. Sui and T. A. Johansen. Moving horizon observer with regularization for detectable nonlinear systems without persistence of excitation. IFAC Symposium on Nonlinear Control, Bologna, 2010.

5. D. Sui and T. A. Johansen. Moving horizon observer with regularization for detectable systems without persistence of excitation. Int. J. Control, 84:1041-1054, 2011.

6. P. E. Moraal and J. W. Grizzle. Asymptotic observers for detectable and poorly observable systems. In IEEE Conf. Decision and Control, New Orleans, pages 109-114, 1995.

7. D.Angeli. A Lyapunov approach to incremental stability properties. IEEE Trans. Automatic Control, 47:410-421, 2002.

8. M. Lazar. Model predictive control of hybrid systems: stability and robustness. $\mathrm{PhD}$ thesis, Eindhoven University of Technology, 2006.

9. R. Abraham, J. E. Marsden, and T. Ratiu. Manifolds, Tensor Analysis, and Applications. Springer-Verlag New York, 1983.

\section{REFERENCES}

1. C. V. Rao, J. B. Rawlings, and D. Q. Mayne. Constrained state estimation for nonlinear discretetime systems: Stability and moving horizon approximation. IEEE Trans. Automatic Control, 48:246-258, 2003. 